\begin{tabular}{|c|c|c|}
\cline { 2 - 3 } & REVISTA SABERES APUDEP & Volumen 3 Número 1 \\
\hline
\end{tabular}

\title{
DIVERSIDAD Y ESTADO DE CONSERVACIÓN DE PECES ARRECIFALES EN PLAYA LA ANGOSTA, PROVINCIA DE COLÓN (PANAMÁ)
}

\author{
DIVERSITY AND CONSERVATION STATE OF REEFFISH FISHES IN PLAYA LA \\ ANGOSTA, PROVINCE OF COLÓN (PANAMÁ)
}

Melo, Nemesio ${ }^{1}$, De León, Yhaidelice ${ }^{1}$, Jorge Morales ${ }^{2}$ y Mónica Contreras ${ }^{1}$

1 Universidad de Panamá Centro Regional Universitario de Colón. Escuela de Biología. Email: nemesiomelo13@gmail.com, Yhaidelicedeleonj@gmail.com monicanuzhat@gmail.com iD orcid.org/0000-0003-0972-6951

2 Smithsonian Tropical Research Institute. Email: jorgemorales0709@gmail.com

\section{RESUMEN}

Los arrecifes coralinos son considerados ambientes dinámicos y de gran importancia biológica debido a la alta diversidad de organismos que interactúan con este ecosistema. Los peces, que son parte de esta diversidad, actúan como controladores ambientales a través de la depredación, el herbivorismo y otros aspectos vinculados a las actividades tróficas. En el área del Caribe, se reporta el declive de comunidades de peces, indicándose entre sus amenazas: la sobrepesca, contaminación, explotación y cambio climático. El objetivo de estudio es determinar la riqueza, abundancia y estado de conservación de la ictiofauna en zonas arrecifales de Playa La Angosta, Colón. Se realizaron 7 muestreos diurnos entre los meses de octubre y diciembre del 2018, en zonas arrecifales de playa La Angosta, se establecieron dos sitios de estudio y dos transectos en banda, utilizándose el método de censo visual de buceo. Se determinó el estado de conservación a partir de la Lista Roja publicada por UICN. Se registró un total de 3668 individuos en 43 especies, 21 familias y 8 órdenes. Las familias más diversas son Haemulidae y Labridae con 6 especies cada una, seguido de Pomacentridae con 4 especies, Scaridae, Lutjanidae, Chaetodontidae y Carangidae con 3 especies. La familia más abundante fue la familia Labridae con un total 1274 individuos que representa un $34.7 \%$ de los taxa estudiadas. Se determinó que Lutjanus synagris está en categoría NT. Es el primer estudio en esta zona que reporta datos sobre diversidad y estado de conservación de peces arrecifales Se recomienda establecer estrategias para conservar tanto la diversidad de peces como cobertura de coral vivo.

PALABRAS CLAVE: Peces arrecifales, Playa La Angosta, Censo Visual De Buceo. 


\begin{tabular}{|c|c|c|}
\cline { 2 - 3 } & REVISTA SABERES APUDEP & Volumen 3 Número 1 \\
\hline
\end{tabular}

\begin{abstract}
Coral reefs are considered dynamic environments of great biological importance due to the high diversity of organisms that interact with this ecosystem. Fish, which are part of this diversity, act as environmental controllers through predation, herbivorism and other aspects linked to trophic activities. In the Caribbean area, the decline of fish communities is reported, indicating among its threats: overfishing, pollution, exploitation and climate change. The objective of the study is to determine the Wealth, Abundance and Conservation Status of the ichthyofauna in reef areas of Playa $\mathrm{La}$ Angosta, Colón. A description of the benthic coverage was made using the Quadrata technique; for the record of wealth and abundance of fish. Seven day samples were carried out between the months of October and December of 2018, in reef areas of La Angosta beach, four band transects were established, using the method of visual diving census. The Conservation Status was determined from the Red List published by IUCN. A total of 3668 individuals were registered in 43 species, 27 genera and 21 families. The most diverse families are Haemulidae and Labridae with 6 species each, followed by Pomacentridae with 4 species, Scaridae, Lutjanidae, Chaetodontidae and Carangidae with 3 species. The most abundant family was the Labridae family with a total of 1274 individuals representing $34.7 \%$ of the taxa studied. It was determined that Lutjanus synagris is in the NT category. It is the first study in this area that reports data on Diversity and Conservation Status of reef fish. It is recommended to establish strategies to conserve both fish diversity and live coral cover.
\end{abstract}

KEYWORDS: Reef fish, La Angosta Beach, Visual Census Diving.

\title{
INTRODUCCIÓN
}

Los arrecifes coralinos son considerados ambientes dinámicos y de gran importancia biológica debido a la alta diversidad de organismos que interactúan con este ecosistema, donde los peces representan un papel particularmente importante para la dinámica de este entorno (Ruiz et al., 2003).

Este ecosistema acoge una gran variedad de especies de peces, siendo estos organismos uno de los grupos más reconocidos, diversos y mejor estudiados en este tipo de ecosistemas. La heterogeneidad espacial que proporcionan estos ambientes ha sido considerada como uno de los factores más importantes que influyen sobre la 


\begin{tabular}{|c|c|c|}
\cline { 2 - 3 } & REVISTA SABERES APUDEP & Volumen 3 Número 1 \\
\hline
\end{tabular}

diversidad de especies de peces, ya que permite una mayor disponibilidad de recursos como refugio y alimento, requisitos que son claves para su supervivencia y reproducción (Choat, 1991; Jones \& Syms, 1998).

Los peces son controladores ambientales: la depredación, el herbivorismo y otros aspectos vinculados a las actividades tróficas; además, la importancia de los peces es reforzada porque forman parte de unos de los principales recursos pesqueros (Freon \& Misund, 1999). Debido al gran impacto antropogénico que se está dando en estos ecosistemas en diversas partes del mundo, resultan importantes las evaluaciones precisas de la composición y abundancia de las comunidades asociadas a estos ambientes. Son pocos los estudios publicados en la provincia de Colón de la república de Panamá, en particular la Costa Arriba, que analicen el estado actual de diversidad y estado de conservación de la ictiofauna asociada a los arrecifes de coral; dado este vacío de información, se justifica el desarrollo de esta investigación, la cual puede contribuir con el registro de especies de interés ecológico para el ecosistema, ya que una línea base de información permitirá establecer estrategias para conservación y regulación de actividades antropogénicas en playas con cobertura coralina. Así como también, identificar cambios en la cobertura de los arrecifes de coral.

\section{MATERIALES Y MÉTODOS}

Este estudio de corte descriptivo, no paramétrico, transversal, se realizó entre los meses de Agosto 2018 y Junio 2019. El sitio de estudio está ubicado en Playa La Angosta corregimiento de María Chiquita distrito de Portobelo, provincia de Colón. Se

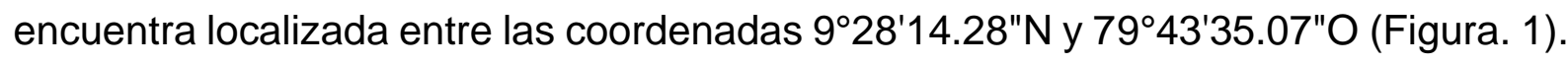



REVISTA SABERES APUDEP
ISSN L 2644-3805

Acceso Abierto. Disponible en:
Volumen 3 Número 1

Enero-Junio 2020

Recibido: 08/08/2019

Aceptado: 06/11/2019

pp.1-10

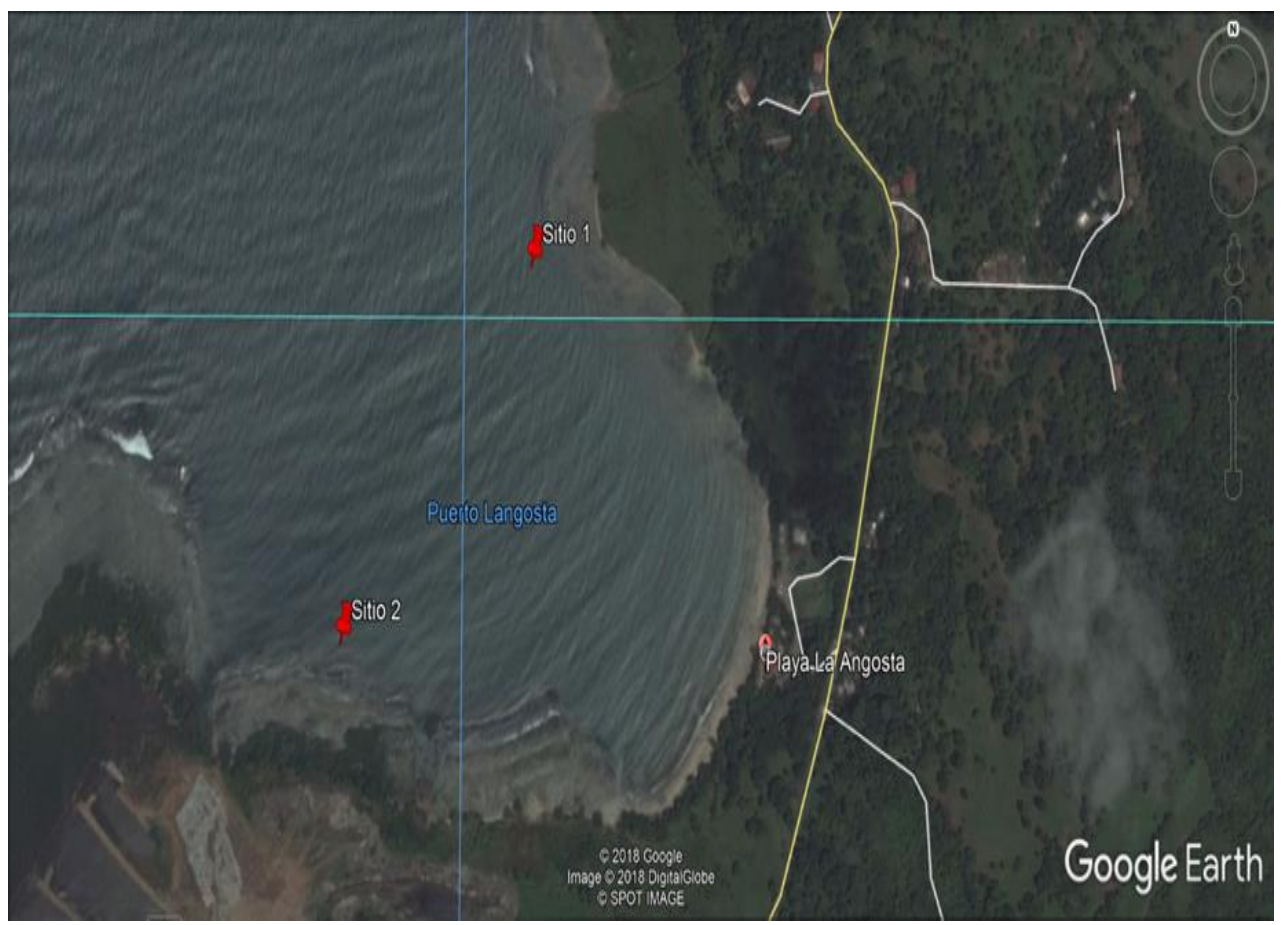

Figura. 1. Vista satelital Playa La Angostacoordenadas 9²8'14.28"N y $79^{\circ} 43^{\prime} 35.07 "$. Fuente. Google Earth.

Para la identificación de la ictifauna, se realizaron siete muestreos diurnos (1 por semana) entre los meses de octubre a diciembre (estación lluviosa) del 2018, se muestreo en 2 sitios en zonas arrecifales de profundidades someras, entre 1 y $3 \mathrm{~m}$, de Playa La Angosta. Se establecieron dos transectos lineales en cada sitio de $15 \mathrm{~m}$ de longitud, delimitado con varillas de hierros incrustadas en el sustrato y se anotaron los individuos que estuvieron a $2 \mathrm{~m}$ cerca del transecto. Figura 2. 
REVISTA SABERES APUDEP

ISSN L 2644-3805

Acceso Abierto. Disponible en:

https://revistas.up.ac.pa/index.php/saberes_apudep
Volumen 3 Número 1

Enero-Junio 2020

Recibido: 08/08/2019

Aceptado: 06/11/2019

pp.1-10

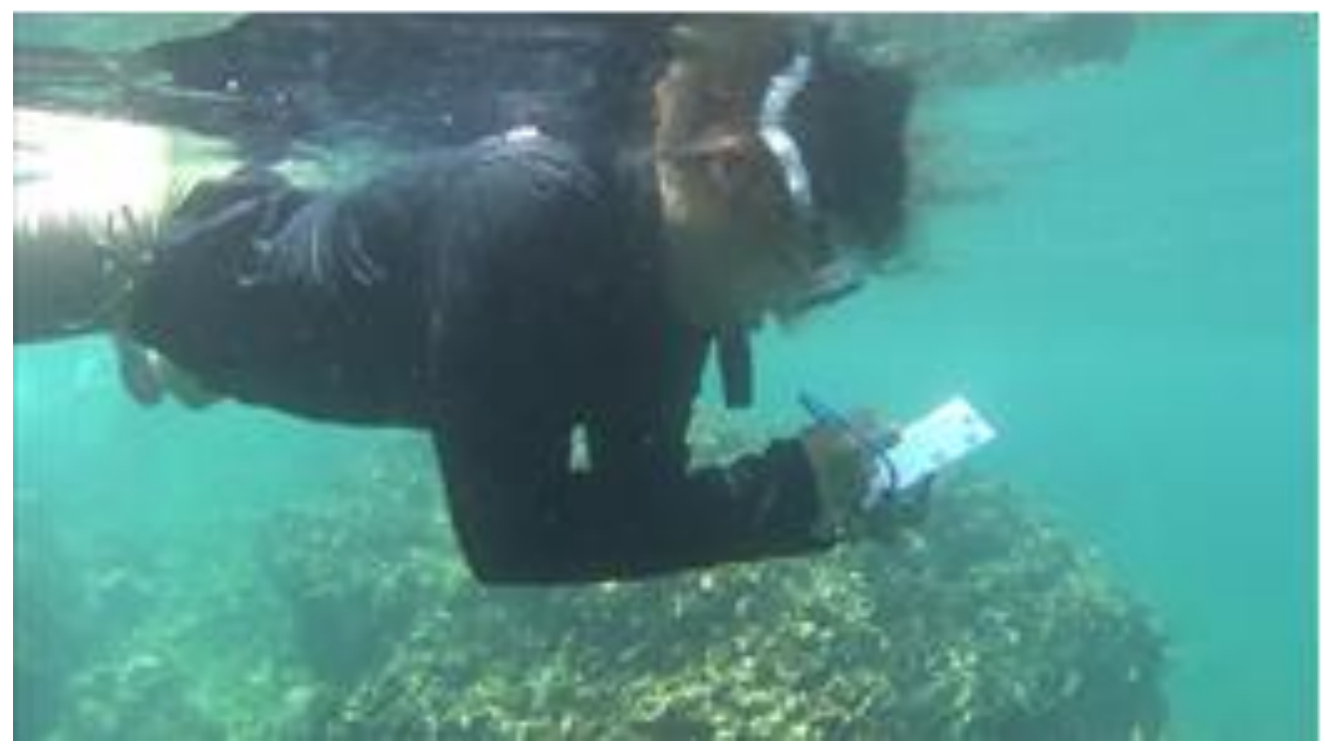

Figura 2. Censo visual de buceo para la identificación de peces arrecifales Playa La Angosta.

Comunidad Ictiofaunistica en Playa La Angosta

Se anotó cada individuo por especie lo que proporcionó un listado de peces y estimaciones de la abundancia relativa de cada especie (Schmitt y Sullivan, 1996). Los apuntes se colocaron en una tabla de buceo mientras que se tomaba videos y fotos para tener una evidencia visual de los especímenes observados y estos se identificaron por medio de la guía de Paul Humann (1994). Se determinó el Estado de Conservación a partir de la Lista Roja publicada por UICN.

\section{RESULTADOS Y DISCUSION}

En la Tabla 1 se presenta el registro de un total de 3668 individuos en 43 especies, 21 familias y 8 Ordenes. 


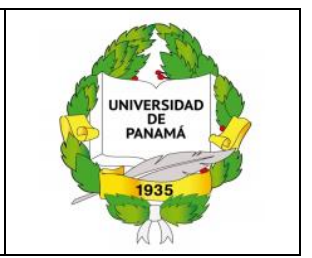

Tabla1 1. Riqueza y Abundancia de Peces Arrecifales, Playa La Angosta, Colón (Panamá)

\begin{tabular}{|c|c|c|c|}
\hline Orden & Familia & Especie & Abundancia \\
\hline Rajiformes & Dasyatidae & Dasyatis americana Hildebrand \& Schroeder, 1928. & 3 \\
\hline Anguilliformes & Muraenidae & Gymnothorax funebris Ranzani, 1839. & 1 \\
\hline Atheriniformes & Atherinidae & Atherinomorus stipes Müller y Troschel, 1848. & +100 \\
\hline Beloniformes & Belonidae & Tylosurus crocodilus Péron \& Lesueur, 1821. & 5 \\
\hline Beryciformes & Holocentridae & Neoniphon vexillarium (Poey, 1860) & 31 \\
\hline Clupeiformes & Clupeidae & Harengula clupeola Cuvier, 1829. & +100 \\
\hline \multirow[t]{34}{*}{ Perciformes } & \multirow[t]{2}{*}{ Acanthuridae } & Acanthurus chirurgus Bloch, 1787. & 32 \\
\hline & & Acanthurus coeruleus Bloch \& Schneider, 1801. & 24 \\
\hline & \multirow[t]{3}{*}{ Carangidae } & Carangoides bartholomaei (Cuvier, 1833) & 2 \\
\hline & & Carangoides ruber (Bloch, 1793) & 9 \\
\hline & & Caranx latus Agassiz, 1831 & 3 \\
\hline & Chaenopsidae & Acanthemblemaria rivasi J. S. Stephens, 1970. & 10 \\
\hline & \multirow[t]{3}{*}{ Chaetodontidae } & Chaetodon capistratus Linnaeus, 1758. & 60 \\
\hline & & Chaetodon ocellatus Bloch, 1787. & 8 \\
\hline & & Chaetodon striatus Linnaeus, 1758. & 1 \\
\hline & \multirow[t]{6}{*}{ Haemulidae } & Anisotremus virginicus Linnaeus, 1758. & 7 \\
\hline & & Haemulon aurolineatum Cuvier, 1830. & 3 \\
\hline & & Haemulon bonariense Cuvier, 1830. & 1 \\
\hline & & Haemulon carbonarium Poey, 1860. & 1 \\
\hline & & Haemulon flavolineatum Desmarest, 1823. & 13 \\
\hline & & Haemulon macrostomum Günther, 1859. & 91 \\
\hline & Kyphosidae & Kyphosus sectatrix Linnaeus, 1758. & 8 \\
\hline & \multirow[t]{6}{*}{ Labridae } & Bodianus rufus Linnaeus, 1758. & 1 \\
\hline & & Halichoeres bivittatus Bloch, 1791. & 99 \\
\hline & & Halichoeres maculipinna Müller y Troschel, 1848. & 15 \\
\hline & & Halichoeres poeyi Steindachner, 1865. & 37 \\
\hline & & Halichoeres radiatus Linnaeus, 1758. & 12 \\
\hline & & Thalassoma bifasciatum Bloch, 1791. & 1110 \\
\hline & Labrisomidae & Labrisomus nисhipinnis Quoy \& Gaimard, 1824. & 2 \\
\hline & \multirow[t]{3}{*}{ Lutjanidae } & Lutjanus apodus Walbaum, 1792. & 21 \\
\hline & & Lutjanus synagris Linnaeus, 1758. & 2 \\
\hline & & Ocyurus chrysurus Bloch, 1791. & 5 \\
\hline & Mullidae & Pseudupeneus maculatus Bloch, 1793. & 1 \\
\hline & \multirow[t]{4}{*}{ Pomacentridae } & Abudefduf saxatilis Linnaeus, 1758. & 616 \\
\hline & & Microspathodon chrysurus Cuvier, 1830. & 220 \\
\hline & & Stegastes adustus Troschel, 1865. & 694 \\
\hline & & Stegastes planifrons Cuvier, 1830. & 175 \\
\hline & \multirow[t]{3}{*}{ Scaridae } & Scarus iseri Bloch, 1789. & 165 \\
\hline & & Sparisoma chrysopterum Bloch \& Schneider, 1801. & 129 \\
\hline & & Sparisoma viride Bonnaterre, 1788. & 40 \\
\hline \multirow[t]{3}{*}{ Tetraodontiformes } & Balistidae & Canthidermis sufflamen Mitchill, 1815. & 2 \\
\hline & Monocanthidae & Cantherhines pullus Ranzani, 1842. & 1 \\
\hline & Tetraodontidae & Canthigaster rostrata Bloch, 1786. & 12 \\
\hline
\end{tabular}




\begin{tabular}{|c|c|c|}
\cline { 2 - 3 } & REVISTA SABERES APUDEP & Volumen 3 Número 1 \\
\hline
\end{tabular}

Las familias de peces arrecifales más diversas son Haemulidae y Labridae con 6 especies cada una, seguido de Pomacentridae con 4 especies, Scaridae, Lutjanidae, Chaetodontidae y Carangidae con 3 especies. Las familias más diversas son Haemulidae, Labridae y Pomacentridae, esto puede deberse a la estrecha relación que existe entre las especies de estas familias con los ecosistemas arrecifales; que utilizan como sitio de alimentación, refugio y criadero. (Figura 3).

El Thalassoma bifasciatum representó un total de 1110 individuos siendo así la especie más abundante esto puede deberse a que los juveniles de esta especie habitan en profundidades someras, también porque son especies con muy pocos depredadores naturales, no son de interés comercial por ende no son afectados por la pesca local; además que estos presentan un método para mantener su población estable cambiando de sexo cuando esta la requiere, y debido a su tamaño y morfología difícilmente son afectados por redes de arrastre.

Se determinó 1 especie en la categoría (NT) y 40 especies en la categoría (LC) De acuerdo con la Lista Roja de la UICN. El Lutjanus synagris (categoría casi amenazado, NT), es una de las principales especies pesqueras a nivel artesanal en la comunidad, por su valor comercial y cultural, y por ser una de las principales especies apetecidas por el sector turístico (Chasqui, 2017). 


\begin{tabular}{|c|c|c|}
\cline { 2 - 3 } & REVISTA SABERES APUDEP & Volumen 3 Número 1 \\
\hline
\end{tabular}
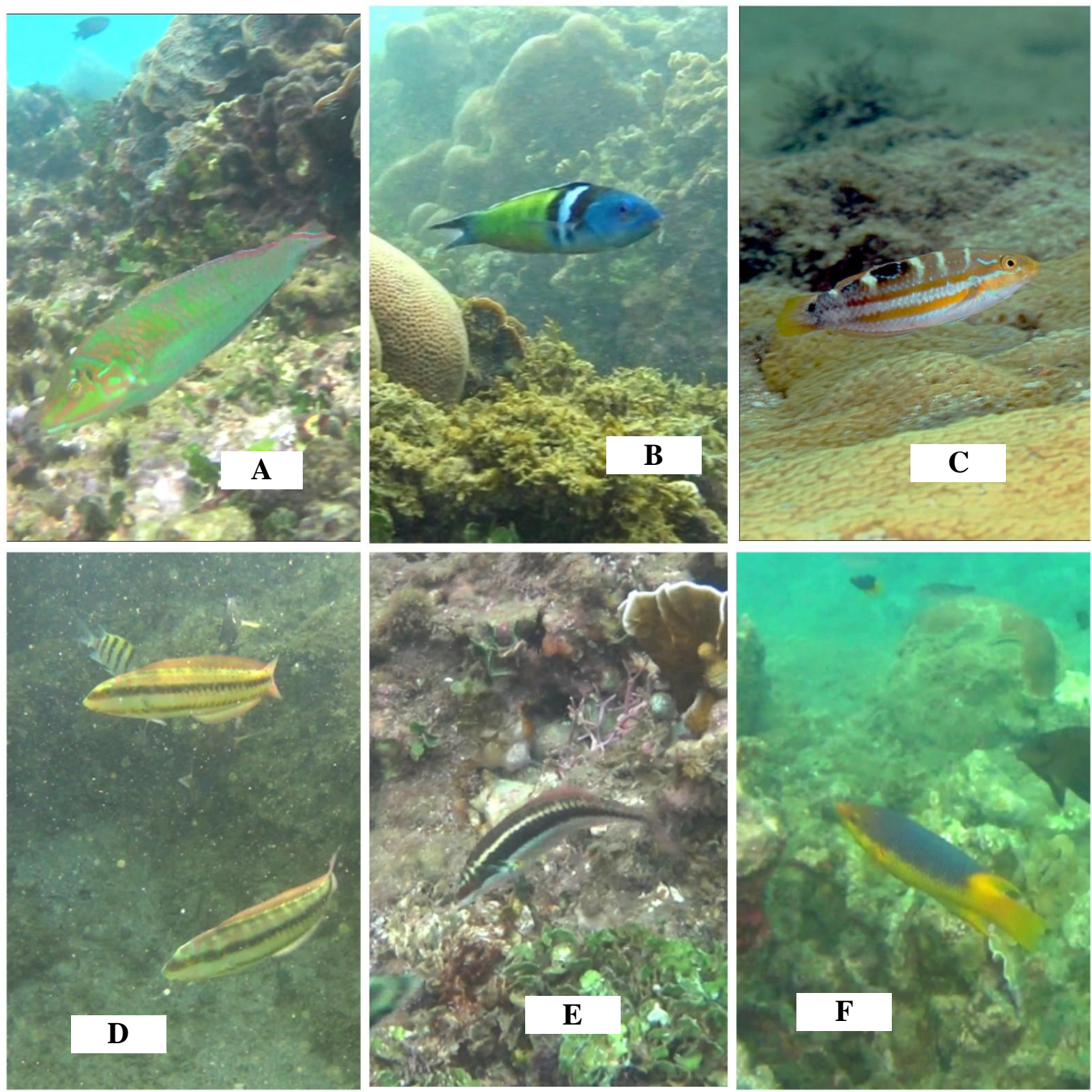
Figura. 3. Representantes
B Thalassoma bifasciatum, C Halichoeres radiatus,
A Halichoeres poeyi, E Halichoeres maculipinna, F Bodianus rufus.
D Halichoeres bivittatus, 


\begin{tabular}{|c|c|c|}
\cline { 2 - 3 } & REVISTA SABERES APUDEP & Volumen 3 Número 1 \\
\hline
\end{tabular}

\section{CONCLUSIÓN}

Este es el primer estudio que da un reporte de datos sobre riqueza y abundancia de peces de arrecifes en playa La Angosta, Colón; estos datos mostraron que existe una alta diversidad de peces arrecifales y de acuerdo con la abundancia se encuentra en un ecosistema cerrado sostenible.

\section{REFERENCIAS BIBLIOGRÁFICAS}

Acero, A y J. Garzón-Ferreira. (1987). Peces arrecifales de la región de Santa Marta (Caribe colombiano). I. Lista de especies y comentarios generales. Acta Biol. Col., 1 (3): 83-105.

Alevizon, W., Richardson, R., Pitts, P. and Serviss, G. (1985). Coral zonation and patterns of community structure in Bahamian reef fishes. Bull. Mar. Sci., 36(2): 304-318.

Booth, D. and Brosnan, D. (1995). The role of recruitment dynamics in rocky shore and coral reef fish communities. Adv. Ecol. Res., 26: 309-385.

Choat, J. (1991). The biology of herbivorous fishes on coral reefs, p. 120-155. In: P. Sale. The Ecology of Fishes on Coral Reefs. Academic, San Diego, USA.

Freon, P. \& Misund, O. A. (1999). Dynamics of Pelagic Fish Distribution and Behaviour: Effects on Fisheries and Stock Assessment. Oxford, Inglaterra: Fishing New Books.

Humann, P. (1996). Reef Fish Identification. Jacksonville, Florida. States United. New World Publications, Inc.

IUCN. Red List $\quad$ Threatened $\quad$ Species. https://www.iucn.org/es/regiones/am\%C3\%A9rica-del-sur/nuestrotrabajo/pol\%C3\%ADticas-de-biodiversidad/lista-roja-de-uicn 


\begin{tabular}{|c|c|c|}
\cline { 2 - 3 } & REVISTA SABERES APUDEP & Volumen 3 Número 1 \\
\hline
\end{tabular}

Ruiz, L., E. Méndez, A. Prieto, B. Marín \& A. Fariña. (2003). Composición, abundancia, y diversidad de peces arrecífales en dos localidades del Parque Nacional Mochima, Venezuela. Cienc. Mar. 29: 185-195.

Schmitt EF, Sullivan KM (1996) Analysis of a volunteer methodfor collecting fish presence and abundance data in the FloridaKeys. Bull Mar Sci 59(2):404-416

Vega-Sequeda, J. 2006. Formaciones coralinas del área de Santa Marta: estado y patrones de distribución espacial de la comunidad bentónica. Tesis Biol. Mar., Univ. Jorge Tadeo Lozano, Santa Marta. 